\title{
Bureau d'expertises extrajudiciaires de la FMH - rapport annuel 2011
}

\section{Lucia Rabia, Valérie Rothhardt}

Avocates, service juridique de la FMH

Correspondance: Bureau d'expertises extrajudiciaires de la FMH Case postale 6159 CH-3001 Berne Tél. 0313591210 Fax 0313591212 lex[at]fmh.ch
En 2011, le Bureau d'expertises extrajudiciaires de la FMH a procédé à l'établissement de 77 expertises. Les experts ont conclu à une ou plusieurs fautes de traitement dans 34 cas et n'ont constaté aucune faute dans 43 autres cas.

Le Bureau d'expertises de la FMH n'est pas compétent pour tous les litiges. Sa tâche est de mandater une expertise lorsque le patient, soigné en Suisse, présume que le médecin exerçant en pratique privée ou à l'hôpital a commis une faute de diagnostic et/ ou de traitement qui a conduit à une atteinte considérable à sa santé et lorsque, de surcroît, le patient n'a pu trouver d'accord sans expertise avec l'assureur responsabilité civile du médecin ou de l'hôpital. Une autre condition est qu'aucun tribunal n'a été saisi du litige ni n'a prononcé de jugement à ce sujet. Depuis l'entrée en vigueur du nouveau règlement en 2002, les informations détaillées sur l'histoire du cas que nous recevons du patient, du médecin/de son assureur ou de l'hôpital permettent de mieux saisir les problèmes à examiner. Le Bureau d'expertises extrajudiciaires de la FMH peut ainsi octroyer plus sûrement son mandat d'expertise à l'équipe d'experts adéquate pour le cas en présence. En outre, la complexité de nombreux cas est d'emblée reconnaissable: dans bien des situations, l'équipe d'experts a dû être composée de représentants de deux ou trois disciplines médicales différentes.

\section{Statistiques du Bureau d'expertises pour l'année 2011}

\section{Méthode}

Durant l'exercice écoulé, 77 expertises ont été menées à bien alors que leur nombre s'était élevé à 81 durant l'exercice précédent. Dans pratiquement un tiers des cas examinés, il s'est agi d'analyser exclusivement des traitements prodigués par des médecins en cabinet privé. Quant au reste des cas (les deux tiers), ils ont porté soit exclusivement sur l'analyse de traitements hospitaliers, soit sur des traitements impliquant conjointement des cabinets privés et des hôpitaux. Les expertises réalisées en 2011 ont nécessité l'intervention de 22 équipes multidisciplinaires d'experts.

En cas d'expertise multidisciplinaire, la classification s'effectue selon la discipline la plus touchée par le cas. Exemple: s'il est fait appel à une équipe d'experts principalement en gynécologie et secondaire- ment en anesthésiologie et qu'une faute est reconnue en gynécologie, et non pas en anesthésiologie, l'expertise sera classée dans la catégorie "gynécologie, faute constatée». Si, dans le même cas, une faute est reconnue en anesthésiologie, et non pas en gynécologie, l'expertise sera classée exclusivement dans la catégorie «anesthésiologie, faute constatée». Si une faute est reconnue dans ces deux disciplines, l'expertise apparaît dans la statistique sous «gynécologie, faute constatée».

La statistique reflète ainsi le résultat déterminant pour le patient et non pas la mesure du travail total fourni par les experts.

\section{Causalité entre la faute et le dommage à la santé}

La réponse à la question de savoir si une faute a été commise dans le diagnostic ou le traitement ne clôt pas l'expertise dans chaque cas. Si des fautes ont effectivement été constatées, il convient alors de déterminer si elles sont aussi à l'origine du dommage à la santé évoqué par le patient. Le droit de ce dernier à une réparation n'existe qu'à partir du moment où la causalité entre la faute et le dommage est reconnue.

Les cas dans lesquels les experts constatent certes une faute, mais pas de rapport de causalité entre celle-ci et le dommage, sont relativement nombreux. $\mathrm{Ou}$, formulé de manière positive: en médecine comme ailleurs, les fautes n'ont pas toutes, heureusement, des conséquences négatives ou graves. L'expert doit donc s'exprimer sur l'état de santé qui serait celui du patient si la faute en question ne s'était pas produite.

La statistique établie depuis des années ne recense pas ce critère de manière explicite. Pour 2011, le lien de causalité entre la faute constatée et le dommage a été clairement reconnu ou reconnu comme étant très probable dans environ un tiers des dossiers concluant à l'existence d'une faute. Toutefois, dans le reste des cas avec fautes avérées, la causalité a été rejetée ou le lien entre faute et dommage a été considéré uniquement comme possible. Il est souvent difficile de quantifier l'influence d'un seul facteur, par exemple d'une faute de traitement, sur le résultat global insatisfaisant. Souvent, d'autres facteurs déterminants influencent le résultat, comme un pronostic de guérison préalablement défavorable dans tel cas particulier, ou des maladies supplémentaires. 
Information médicale au patient et communication entre médecin et patient

L'information médicale au patient à elle seule ne peut pas faire l'objet d'une expertise de la FMH. Elle peut toutefois être abordée parallèlement à la faute de diagnostic et/ou de traitement supposée. De manière tout à fait générale, nous aimerions par expérience souligner combien il est important que cette information du patient soit suffisamment documentée.

\section{Tableau 1}

Vue d'ensemble globale 1982-2011.

\begin{tabular}{|c|c|c|c|c|}
\hline & Expertises établies & $\begin{array}{l}\text { Fautes de diagnostic et } \\
\text { de traitement avérées }\end{array}$ & $\begin{array}{l}\text { Fautes de diagnostic et } \\
\text { de traitement niées }\end{array}$ & $\begin{array}{l}\text { Fautes de diagnostic et } \\
\text { de traitement indéterminées }\end{array}$ \\
\hline Toute la Suisse 1982-2010 & 3314 & 1106 & 2112 & 96 \\
\hline Suisse alémanique et Tessin 2011 & 52 & 25 & 27 & 0 \\
\hline Suisse romande 2011 & 25 & 9 & 16 & 0 \\
\hline Toute la Suisse 2011 & $77(100 \%)$ & $34(44,2 \%)$ & $43(55,8 \%)$ & $0(0,0 \%)$ \\
\hline Total 1982-2011 & $3391(100 \%)$ & $1140(33,6 \%)$ & $2155(63,6 \%)$ & $96(2,8 \%)$ \\
\hline Total des 10 dernières années 2002-2011 & $804(100 \%)$ & $357(44,4 \%)$ & $432(53,7 \%)$ & $15(1,9 \%)$ \\
\hline
\end{tabular}

Tableau 2

Résultats par spécialité 1982-2011.

\begin{tabular}{|c|c|c|c|c|}
\hline & Expertises établies & $\begin{array}{l}\text { Fautes de diagnostic et } \\
\text { de traitement avérées }\end{array}$ & $\begin{array}{l}\text { Fautes de diagnostic et } \\
\text { de traitement niées }\end{array}$ & $\begin{array}{l}\text { Fautes de diagnostic et } \\
\text { de traitement indéterminées }\end{array}$ \\
\hline Médecine générale & 238 & 87 & 141 & 10 \\
\hline Anesthésiologie & 118 & 38 & 77 & 3 \\
\hline Chirurgie & 824 & 289 & 508 & 27 \\
\hline Dermatologie & 29 & 9 & 18 & 2 \\
\hline Gastro-entérologie & 14 & 2 & 12 & 0 \\
\hline Gynécologie et obstétrique & 425 & 162 & 256 & 7 \\
\hline Chirurgie de la main & 51 & 19 & 30 & 2 \\
\hline Chirurgie cardiaque et vasculaire thoracique & 25 & 8 & 16 & 1 \\
\hline Médecine interne & 226 & 71 & 151 & 4 \\
\hline Cardiologie & 20 & 12 & 8 & 0 \\
\hline Chirurgie maxillo-faciale & 23 & 3 & 20 & 0 \\
\hline Chirurgie pédiatrique & 14 & 4 & 10 & 0 \\
\hline Psychiatrie pédiatrique & 1 & 0 & 1 & 0 \\
\hline Néphrologie & 2 & 0 & 2 & 0 \\
\hline Neurochirurgie & 89 & 24 & 63 & 2 \\
\hline Neurologie & 24 & 7 & 16 & 1 \\
\hline Oncologie & 8 & 4 & 4 & 0 \\
\hline Ophthalmologie & 132 & 39 & 87 & 6 \\
\hline Chirurgie orthopédique & 636 & 232 & 389 & 15 \\
\hline Oto-rhino-laryngologie ORL & 118 & 27 & 87 & 4 \\
\hline Pédiatrie & 67 & 27 & 37 & 3 \\
\hline Pathologie & 6 & 4 & 2 & 0 \\
\hline Pharmacologie & 2 & 2 & 0 & 0 \\
\hline Médecine physique et réadaptation & 13 & 3 & 9 & 1 \\
\hline Chirurgie plastique, reconstructive et esthétique & 126 & 27 & 97 & 2 \\
\hline Pneumologie & 1 & 1 & 0 & 0 \\
\hline Psychiatrie & 15 & 7 & 8 & 0 \\
\hline Radiologie & 51 & 14 & 34 & 3 \\
\hline Radio-oncologie & 1 & 1 & 0 & 0 \\
\hline Rhumatologie & 16 & 5 & 11 & 0 \\
\hline Urologie & 76 & 12 & 61 & 3 \\
\hline Total 1982-2011 & 3391 & 1140 & 2155 & 96 \\
\hline
\end{tabular}


Dans plusieurs cas, les experts sont en effet parvenus à la conclusion qu'il n'y avait pas de faute de diagnostic ni de traitement, mais que l'information aux patients sur le plan médical était insuffisante ou faisait défaut ou qu'elle était lacunaire, voire pas du tout documentée.

A diverses reprises, il s'est aussi avéré que la communication entre médecins et patients laissait à désirer. Si les résultats du traitement ne correspondent pas entièrement aux attentes du patient ou si le traitement prend subitement une mauvaise tournure, une communication insuffisante de la part des médecins risque d'éveiller ou de renforcer chez les patients la présomption qu'une faute a été commise.

\section{Limite de la valeur probante de la statistique}

Le nombre total de 77 expertises terminées en 2011 incite en lui-même à la prudence si l'on veut en tirer des conclusions éventuelles. Cette statistique est peu représentative de la situation en matière de responsabilité civile dans les hôpitaux et chez les médecins en Suisse. Un grand hôpital cantonal non universitaire est confronté, à lui seul, entre vingt et trente cas de responsabilité civile chaque année.

Cette statistique montre donc uniquement combien d'expertises ont été établies dans les diverses disciplines par le Bureau d'expertises de la FMH et dans combien d'entre elles une faute de diagnostic et/ou de traitement a été constatée ou niée. Le petit nombre de données à disposition et le manque de valeurs comparatives ne permettent pas d'en tirer d'autres conclusions. On ne saurait donc, par exemple, procéder sur cette base à des calculs pour établir le pourcentage de fautes par discipline médicale ou dans la médecine en général.

Le pourcentage de fautes reconnues a légèrement reculé par rapport à l'exercice précédent et s'élève à $44 \%$ pour l'année 2011 (contre 50\% en 2010 et 2009). Ici également, la prudence est de mise si l'on veut procéder à une interprétation de ces modifica-

\begin{abstract}
Entretien préliminaire par téléphone, adresses, documents
Depuis des années, le Bureau d'expertises extrajudiciaires de la FMH donne l'occasion aux patients, à leurs avocats et à d'autres conseillers de discuter du cas d'espèce, par téléphone avec le Bureau d'expertises, avant le dépôt définitif de la demande. Où et par qui une faute aurait-elle pu être commise sur la base des recherches préliminaires? Quelles sont les autres causes de fautes possibles? En quoi pourrait consister le dommage à la santé? Quels sont les éléments particuliers que doit indiquer le Bureau d'expertises aux délégués des sociétés de discipline médicale qui proposent des experts? etc. Ces discussions préalables nécessitent peut-être une demi-heure ou une heure, mais elles permettent d'éviter nombre de questions ultérieures et font gagner un temps précieux dans l'intérêt de la procédure d'expertise.
\end{abstract}

Les documents nécessaires au dépôt d'une demande d'expertise peuvent être obtenus auprès du Bureau d'expertises extrajudiciaires de la $\mathrm{FMH}$, Case postale 6159, 3001 Berne, tél. 03135912 10, fax 0313591212.

D'autres informations peuvent être obtenues sous www.fmh.ch $\rightarrow$ Services $\rightarrow$ Bureau d'expertises extrajudiciaires. tions. Il suffit qu'un petit nombre de cas soit bouclé durant l'année précédente, en cours ou suivante et apparaisse dans la statistique pour modifier sensiblement ce pourcentage. Le Bureau d'expertises extrajudiciaires est tenu de procéder avec fair-play et veille à ce que chaque dossier soit traité correctement.

Ce qui n'apparaît pas dans la statistique, c'est toujours le grand investissement de temps et de ressources engagé dans des demandes qui ne conduiront finalement pas à une expertise. Patients, avocats, médecins, assurances et autres institutions s'adressent, avec des questions multiples et variées, au Bureau d'expertises extrajudiciaires qui tente, dans la mesure du possible, de leur donner des informations utiles, même si les problèmes exposés n'entrent pas dans son domaine de compétences. Il est aussi souvent difficile de faire comprendre à un patient que le Bureau d'expertises de la FMH n'offre pas une procédure informelle et unilatérale et que les complications survenues ou les attentes de guérison déçues ne peuvent pas toutes conduire à une expertise.

\section{Assurance-qualité}

L'assurance-qualité revêt une grande importance dans la procédure suivie par le Bureau d'expertises. Les démarches suivantes y contribuent:

- Les sociétés de discipline médicale proposent, pour chaque cas particulier, des experts qui acceptent le mandat confié avec l'accord de l'ensemble des parties concernées. Dès le début, une équipe est constituée avec des experts de différentes disciplines lorsque le cas l'exige. Le but visé est d'assurer une évaluation par des personnes compétentes en la matière, de manière analogue à un traitement médical.

- En outre, le schéma destiné aux experts et utilisé depuis des années s'avère très utile car il les aide dans leur tâche d'élaborer une expertise qui permette aux parties de régler le litige.

- Un instrument particulier de l'assurance-qualité est la relecture du projet d'expertise par le service juridique de la FMH. A quelques exceptions près, les patients approuvent cette manière de procéder. La tâche des deux juristes est ainsi de soutenir les experts dans la rédaction d'une expertise qui soit complète, pertinente et, surtout, compréhensible pour des profanes.

\section{Formation des experts}

Les juristes du Service juridique de la FMH participent régulièrement à des rencontres dont le but est de former les médecins à l'expertise médicale ou qui abordent la question de la responsabilité civile du médecin. Pendant l'exercice sous revue, elles sont à nouveau intervenues lors des formations en expertises médicales de Swiss Insurance Medicine (SIM) et lors du congrès annuel de la Société suisse de médecine interne (SSMI) à Lausanne. 


\section{Durée de la procédure}

La durée de la procédure est régulièrement critiquée, avant tout par les patients qui attendent une réponse rapide à leurs questions. Il n'arrive pas souvent qu'une expertise puisse être close moins d'un an après l'envoi de la demande. D'autre part, une procédure réglementée, transparente et acceptable par tous prend du temps. Selon les cas, la recherche d'experts compétents dure à elle seule très longtemps, en particulier lorsque les experts proposés ne sont pas acceptés par l'une des parties. A cela s'ajoute généralement le temps investi par le Service juridique de la FMH pour relire les projets d'expertises, et le cas échéant, la révision ou l'apport de compléments d'informations par l'expert, ce qui, en contre partie, augmente souvent la compréhension et la pertinence de l'expertise.

Lorsque plusieurs experts ont été mandatés, chaque étape requiert plus de temps, depuis l'audition et l'examen du patient jusqu'à la rédaction finale de l'expertise. Il faut souligner que la charge de travail de nombreux experts est telle qu'ils trouvent à peine le temps nécessaire pour effectuer ce genre de mandats supplémentaires dans le délai souhaité; la plupart y sacrifient même une partie de leurs moments de loisirs.

Certains hôpitaux notamment demandent de plus en plus fréquemment à pouvoir bénéficier d'un traitement de faveur par rapport au Règlement du Bureau d'expertises de la FMH, ce qui entraîne d'importants retards ou rend l'établissement d'une expertise carrément impossible dans certains cas. D'autres institutions ou médecins en revanche signalent clairement qu'ils se soumettent au règlement et qu'ils s'attendent à ce que les autres parties fassent de même. Le Bureau d'expertises extrajudiciaires offre une prestation, mais ne détient pas le monopole. S'il accepte d'établir une expertise, il veut pouvoir diriger la procédure et traiter équitablement toutes les parties.

\section{Conseil scientifique}

Le Conseil scientifique surveille l'activité du Bureau d'expertises extrajudiciaires sur mandat du Comité central de la FMH. Il n'a pas de compétence décisionnelle en ce qui concerne les différents cas traités mais décharge le Comité central de son activité de surveillance et soutient le bureau d'expertises en cas de difficultés lors d'une procédure d'expertise. Au cours de l'exercice sous revue, le Conseil scientifique a tenu deux séances et a examiné par sondage quelques dossiers d'expertise.

Le Conseil scientifique se compose des membres suivants: le Dr Bruno Lerf, président, le Dr Jürg Knessl et Me Massimo Pergolis, avocat.

\section{Personnel}

Responsable du Bureau d'expertises extrajudiciaires, Madame Susanne Friedli se charge des dossiers de la Suisse alémanique et du Tessin. Son suppléant, Monsieur Sébastian Lerch, de langue maternelle française, traite les dossiers de la Suisse romande. Le Bureau d'expertises extra-judiciaires est supervisé par Madame Lucia Rabia, avocate et Madame Valérie Rothhardt, avocate, toutes deux en fonction au Service juridique de la FMH.

\section{Remerciements}

Pour pouvoir fonctionner, le Bureau d'expertises extrajudiciaires a besoin de la collaboration de nombreuses personnes. Nous remercions les sociétés de discipline médicale et leurs délégués de leur précieux soutien ainsi que les experts pour la grande somme de travail accomplie en vue d'éclaircir les cas. Nous remercions les médecins traitants ainsi que les directions d'hôpitaux qui ont coopéré de manière ouverte et correcte à la réalisation d'expertises à la demande des patients.

Mme Susanne Friedli et M. Sébastian Lerch sont chargés du traitement des dossiers, depuis la réception de la première demande jusqu'à l'envoi des rapports d'expertise. Ils sont les interlocuteurs de toutes les parties et fournissent un grand travail de coordination et de conseils. Nous leur adressons ici nos vifs remerciements pour leur engagement. 